\title{
Probe recall and short-term memory: Some evidence for nonlinear search strategies
}

\author{
GILBERT J. HARRIS \\ Herbert H. Lehman College, City University of New York, New York, New York 10468
}

\begin{abstract}
The subjects saw sequences of three, five, or seven digits. A subsequent probe test signaled subjects to respond with the item that had followed (forward probe) or preceded (backward probe) the probe item in the sequence. Regardless of probe direction, correct reaction times increased linearly as a function of list length. Correct reaction time as a function of serial position yielded nonlinear curves, suggesting that subjects did not search memory serially in a forward direction. Instead, the shapes of these functions suggested response strategies which involved multiple entry or anchor points and backward search through short-term memory.
\end{abstract}

In a probe recall task, the subject sees a sequence of items one at a time and is then presented with a test item (probe item). Typically, his task is to recall the item that followed the probe item in the sequence (target item). This technique was originally developed by Murdock (1961) to study the short-term forgetting of individual items. Investigators employing this procedure have been primarily concerned with the probability of recalling the target item correctly as a function of the serial position of the probe item, the number of items preceding the probe item and the number of items following it (e.g., Waugh \& Norman, 1965; Murdock, 1968).

Stemberg (1967) used reaction time as the basic dependent variable in the probe task and found a linear function relating mean reaction time for correct responses to length of sequence. Mean reaction time also increased approximately linearly with increasing serial position for some subjects. These list length and serial position functions were viewed as evidence for a self-terminating process of scanning to locate which proceeded at about four items per second. This self-terminating process implies that the probe item is compared to each of the items stored in short-term memory (STM) until a match is found, at which time some kind of shift to the target item's representation in memory occurs, enabling its retrieval. Given Sternberg's (1967) assumption that the mean comparison time per item and the mean time for the shifting process are independent of both list length and serial position, the linear functions are predicted, if the search through STM begins at the representation of the first item and proceeds through the list serially. The individual subjects in Sternberg's (1967) experiment, however, did not all exhibit linearly increasing superimposed functions relating reaction time to serial position.

The present experiment attempted a partial replication of Stemberg's (1967) functions relating reaction time to list length and serial position in the usual probe recall situation (forward probe). In addition, extensions of the task explored two issues.
First, a serial memory search, beginning at the first stored item, is not always the most efficient strategy. If additional anchor points (Feigenbaum\& Simon, 1962) or entry points to STM are utilized, the subject may be able to search STM in a backward direction. For instance, what if the target item is the item preceding the probe item (backward probe)? Given that only forward order information is stored along with items in STM, the mechanism of shifting to retrieve the target would not be clear. Alternatively, some kind of backward link might also be stored with each item to allow search to proceed from STM Cell $\mathrm{n}$ to Cell $\mathrm{n}-\mathrm{l}$.

Secondly, given an uncertain condition in which the direction of the target item relative to the probe item is withheld from the subject until the probe is presented, STM processing strategies may be affected.

In general, the present study is designed to offer possible explanations for nonlinear search patterns in the probe recall task as manifest by individual serial position functions.

\section{METHOD}

\section{Subjects}

The subjects were six male undergraduate students who volunteered to participate in the experiment.

\section{Design}

Two independent variables, type of target required (forward probe, backward probe, and uncertain probe) and list length (three, five, and seven) were manipulated within subjects. The order of the three conditions of type of target required was counterbalanced across the six subjects one condition per day for 3 days in a Latin square arrangement.

For the forward and backward probe conditions, each serial position at each list length was probed 20 times (except, of course, for the last position for the forward probe and the first position for the backward probe). For the uncertain probe conditions, each serial position at each list length for each probe direction was tested 10 times. Thus, each day's testing contained 240 trials organized into two blocks of 120 each. Within each bluck, 10 instances of each possible combination of serial position and list length were present in random ordor for the forward and backward probe conditions, and 5 instances of each combination of serial position, list length, and probe direction were present for the uncertain probo condition. 


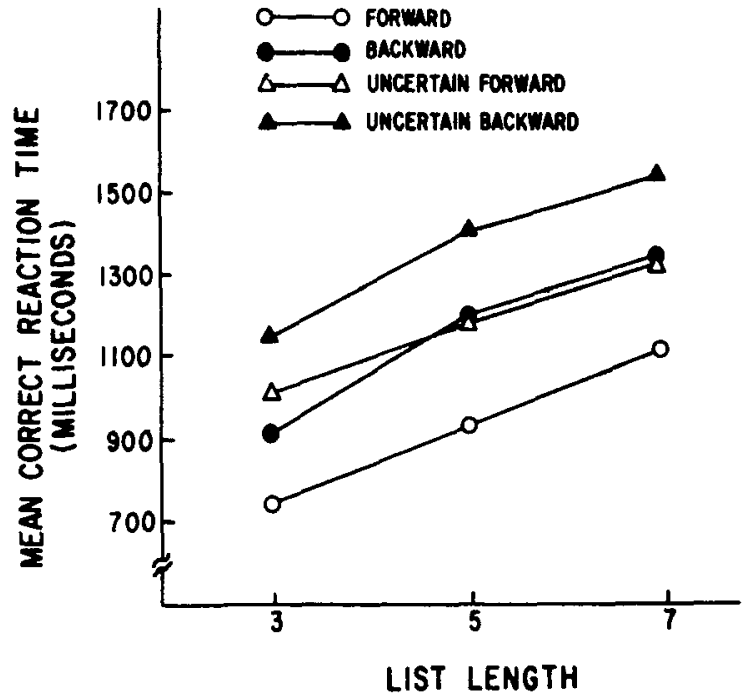

Figure 1. Mean latency of correct response as a function of list length (6 subjects).

On the first day, all subjects received 10 test trials for each of the three probe conditions to acquaint themselves with the apparatus before they embarked on the first day's testing. In addition, 5 unscored practice trials preceded the 240 test trials on each day.

\section{Materials}

All sequences consisted of random, nonrepeated digits from the set 123456789 . The probe digit always appeared in the sequence.

\section{Procedure and Apparatus}

All items were visually presented with a digital decoding display unit (Binaview) and appeared white on a dark background $1 \frac{1 / 2}{2}$ in high and $1 \frac{1 / 4}{4}$ in wide. The unit was driven by Grason-Stadler electronic timing modules, and the sequences were preprogrammed on paper tape which was read by an EECO 5000 photoblock tape reader.

The subjects were tested individually. Each subject sat in a soundproof booth, and a display consisting of the Binaview and two signal lights were on a table directly in front of him. The subject initiated each sequence by pressing a start button also on the table in front of him. The sequence then appeared on the Binaview, one digit at a time, at the rate of $0.6 \mathrm{sec}$ per item (stimulus duration was $0.5 \mathrm{sec}$ ). After the last item of a sequence, a blank dark field appeared for $3.5 \mathrm{sec}$, during which the subject had been instructed to rehearse the sequence in any manner he preferred. The delay from the presentation of the last item to the onset of the probe was used to eliminate the effects of sensory memory. Murdock $(1963 ; 1966)$ measured reaction times in the probed recall of paired associates without employing any such delay and found response latency to be a decreasing function of serial position, indicating that the last several items are stored in some type of "ready access echo box." After the delay the probe appeared along with one of the two signal lights. A green light, above and to the right of the display unit, signified that the target item was that which followed the probe item in the sequence. A yellow light, above and to the left of the display unit, signified that the target item was that which preceded the probe item in the sequence. Thus, for all forward probe sequences, the green light came on with the probe item while the yellow light came on with the probe for all backward probe sequences. In the uncertain probe condition, the probe direction varied from trial to trial. The subject's verbal response automatically removed the probe digit from the display unit and also shut off the signal light. The subject was then ready to initiate the next sequence by pushing the start button.

The reaction time, measured from onset of probe and signal to the outset of the subject's verbal response, was recorded via a Grason-Stadler E7300A-1 voice operated relay and a Hickock DP150 counter accurate to the nearest thousandth of a second. Reaction times were printed by a Hickock PR 4900 printer. Responses were monitored by the experimenter and recorded with a Sony TC105 tape recorder outside the test booth.

\section{RESULTS}

\section{Errors}

All analyses and discussion concerning reaction time are based upon correct responses only. The overall error rate, across all subjects and experimental conditions, was $6.84 \%$. Errors increased with increasing list length but did not vary as a function of probe condition. The error rates were $2.36 \%, 3.75 \%$, and $10.37 \%$ for list lengths three, five, and seven, respectively. These differences were significant, $[F(2 / 10)=21.52, p<.001]$, with a subsequent Newman-Keuls analysis $(\alpha=.01)$ showing that more errors were made at List Length 7 than at the two shorter lengths.

Although percentage of errors decreased slightly on Day 3 (Day 1-7.15\%; Day 2-7.85\%; Day 3-5.49\%), this practice effect was not statistically significant.

Of the 295 errors obtained for all subjects, $61 \%$ were other items in the sequence, $20 \%$ were items not in the sequence, $10 \%$ were naming the probe item, and $9 \%$ were failure to respond or other miscellaneous responses.

\section{Correct Reaction Time as a Function of List Length}

The uncertain-probe condition was divided into its component forward and backward-probe trials for purposes of correct reaction time analyses. As shown in Figure 1, mean reaction time increased as a function of list length for all four probe conditions. Analysis of variance showed this increase to be significant $[F(2 / 10)=40.18, p<.001]$ with a Newman-Keuls post test $(\propto=.01)$ showing differences between all conditions.

The equations of the fitted lines for the four probe conditions are: forward $\mathrm{RT}=502+85 \mathrm{x}$; backward $\mathrm{RT}=614+105 \mathrm{x}$; uncertain forward $\mathrm{RT}=775+77 \mathrm{x}$; uncertain backward RT $=821+107 x$. The $y$-intercept of the fitted line for the backward-probe condition is greater than that for the forward-probe condition. Similarly, the y-intercept of the uncertain-backward condition is greater than for the uncertain-forward condition. In addition, both uncertain-probe conditions have larger $y$-intercepts than do the corresponding pure probe conditions. These differences produced a significant effect of probe condition $[F(3 / 15)=22.66$, $p<.001]$. The slopes of the two backward probe conditions are similar and are both steeper than the two forward probe conditions, whose slopes are also similar 
to each other. These slope differences, however, did not produce a significant interaction between list length and probe condition.

Mean correct reaction time was not affected by day of testing nor did days interact with list length. The mean reaction times across all probe conditions and subjects were $1107 \mathrm{msec}$ for Day 1, $1088 \mathrm{msec}$ for Day 2, and $1132 \mathrm{msec}$ for Day 3.

\section{Correct Reaction Time as a Function of Serial Position}

The shapes of the serial position functions indicated marked individual differences. Subjects 1,2 , and 3 produced similar functions which are combined in Figure 2. The shapes of these curves are monotonically increasing for both probe directions at List Length 3 only. The forward probe conditions yielded a linearly increasing function at List Length 5 , but a drop in the curve for the backward probes obtained at Serial Position 5. The shapes of the curves for both probe directions are identical for List Length 7 , indicating a dip at the fourth data point and another drop at the final point.

Subjects 4, 5, and 6 show idiosyncratic deviations from the curves of Figure 2. The backward probe curves of Subject 4 (Figure 3) show only one dip, at Serial Position 4, rather than the two noted in Figure 2. Subject 5 (Figure 4) shows a dip at Serial Position 3 for

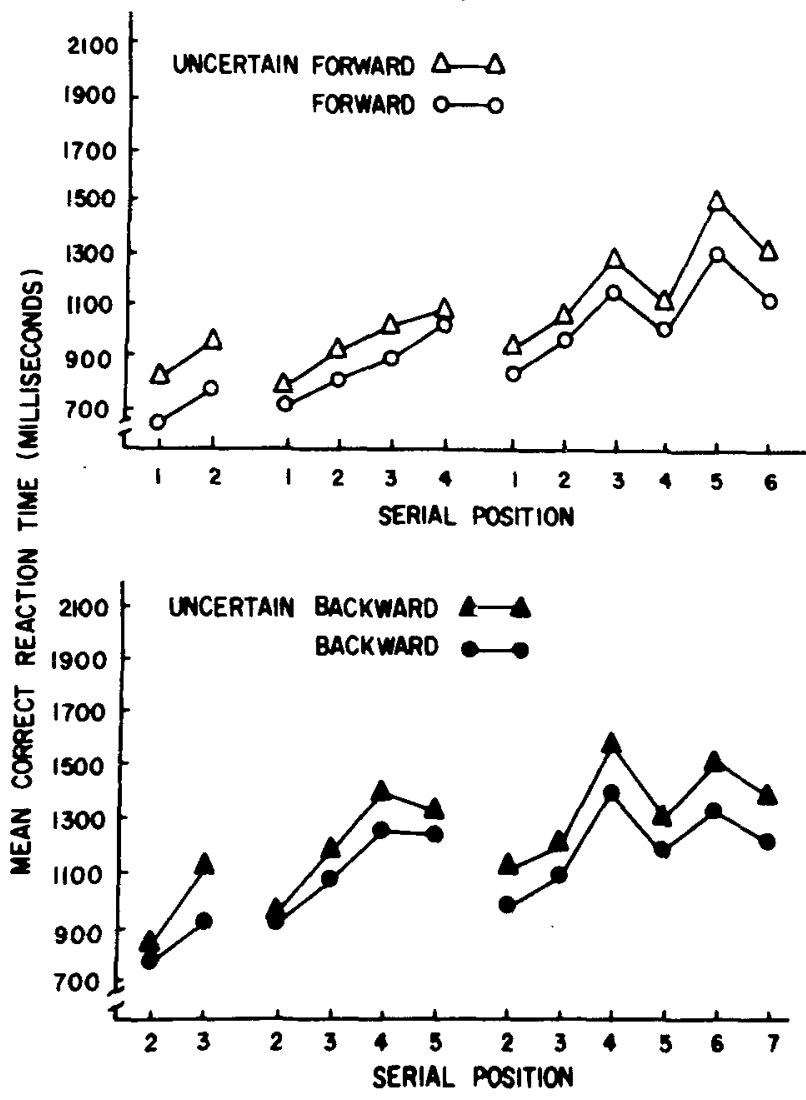

Figure 2. Mean latency of correct responses as a function of serial position of the probe: Subjects 1,2 , and 3 .

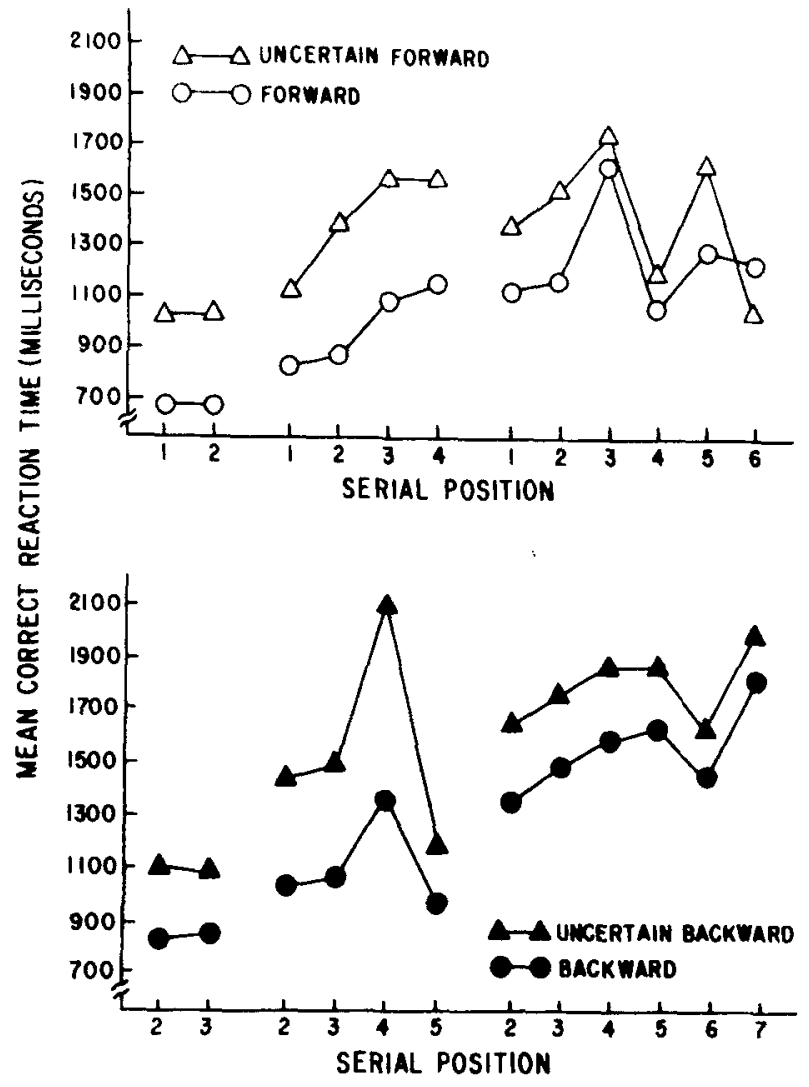

Figure 3. Mean latency of correct responses as a function of serial position of the probe: Subject 4.

List Length 5 in the forward probe curves rather than at Serial Position 4 as noted in Figure 2. Subject 6 (Figure 5) shows only one drop at List Length 7 for both probe directions and this is at the final data point in each case.

It is important to note, however, that all six subjects exhibit within-subject reliability in the shape of the serial position function between their "pure" and corresponding uncertain trials at all list lengths for both probe directions. That is, no statistically significant interactions resulted from the comparisons shown in Figures $2 \cdot 5$.

\section{DISCUSSION}

The increase in mean correct reaction time when probes were backward as opposed to when they were forward suggests that backward link information is in some sense more difficult to retrieve than is forward link information. It seems that for a given list length, the difference in reaction times between the forward and backward conditions results from different shift times for getting from the probe to the target item in memory. This view, which implies that it is easier to search STM in a forward direction (i.e., it takes less time to retrieve a forward link than to retrieve a backward link), has been put forward previously by Yntema and Trask (1963). Possibly, all order information associated with a STM 


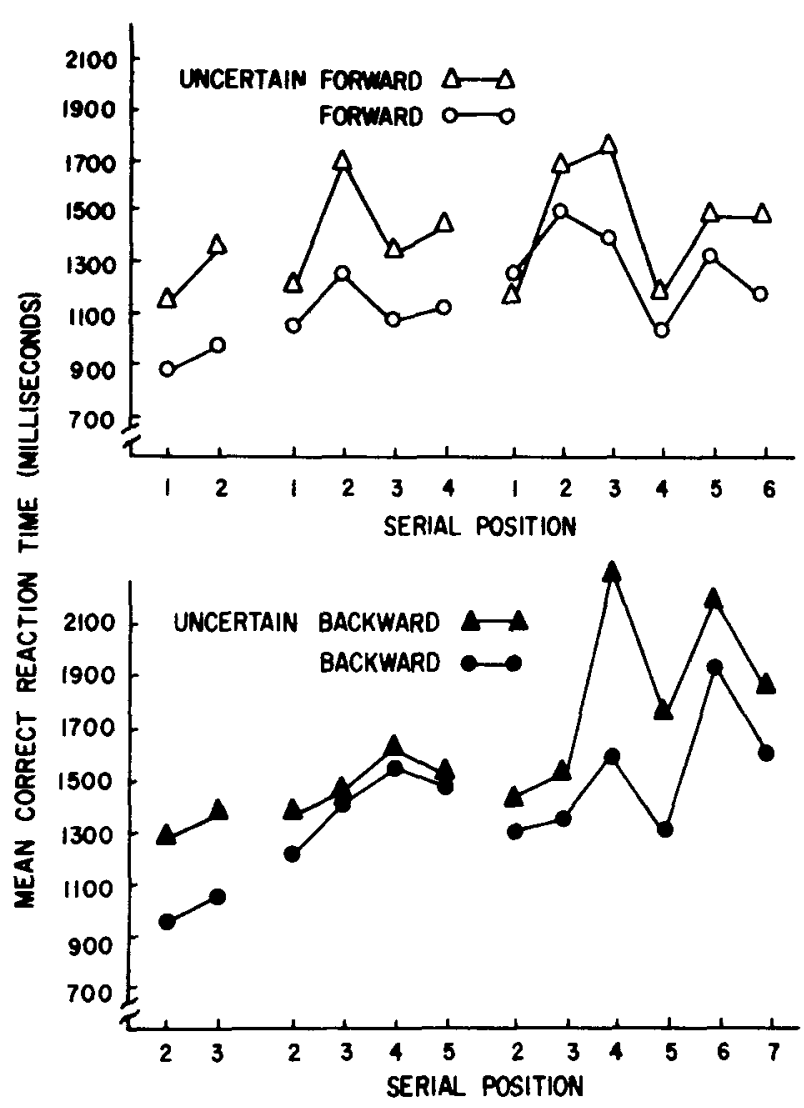

Figure 4. Mean latency of correct responses as a function of serial position of the probe: Subject 5.

cell is stored in a specialized area of that cell with forward order information being located in the most accessible locations, resulting in shorter retrieval times and thus shorter reaction times when forward link information is required.

The effect of uncertainty of direction of the probe upon reaction time did not interact with probe direction, list length, or serial position, suggesting that the information from the light, signaling the subjects as to probe direction, was processed independently of (and previous to) the search through STM to locate the probe item. From Figure 1, the mean time for subjects to input this information is on the order of $200 \mathrm{msec}$.

Clearly, the results of Stemberg (1967) were not fully replicated and his theoretical conclusions concerning the search strategies involved in the probe recall task may be amended by the data obtained in the present experiment. Mean correct reaction times did increase linearly with increasing list length for the forward probe condition. However, the monotonic increase of reaction times as a function of serial position, reported in the earlier study for all list lengths, was obtained in the present study only at List Lengths 3 and 5 . Given the consistency of this finding for all subjects, an alternative explanation is proposed, based on a strategy analysis of the task and the obtained serial position functions. Sternberg's (1967) suggested self-terminating scan to locate implies that subjects typically store the items as they are presented in a unidirectional array which may be entered only at the location of the initial item. Further, the scanning process is serial and in a forward direction only.

An alternative is an STM structure with multiple entry points or anchor points (Feigenbaum \& Simon, 1962) and bidirectional search capability organized by a higher level response strategy process. Verbal protocols solicited from subjects after their participation in the present study provide further insights into the strategies employed. Subjects 1, 2, 3, and 5 reported grouping the digits at List Length 7 in a 3-and 4 "telephone number" arrangement. Grouping a list into two parts resulted in three anchor points into the STM representation of the sequence. The first and seventh items serve as anchor points delineating the beginning and end of the entire sequence in memory while the fourth item serves as a middle anchor point indicating the beginning of the second grouping in memory.

A rapid time-based self-terminating scan for presence is the basis of one possible mechanism for choosing an entry point into STM for a given sequence. In other words, by monitoring the time taken to affirm the presence of the probe item, the subject (i.e., the response strategy process) learns its approximate location in the sequence. Then, depending upon the

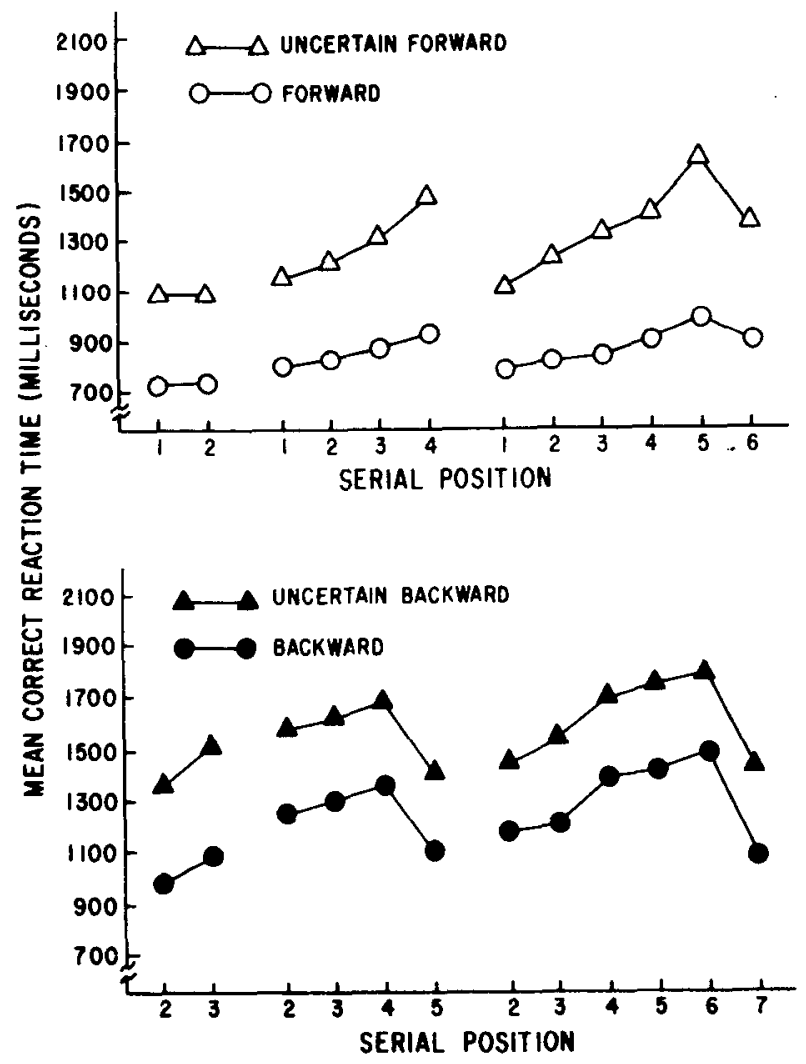

Figure 5. Mean latency of correct responses as a function of serial position of the probe: Subject 6. 
retrieval strategy he is employing, he chooses the appropriate anchor point at which to initiate his scan to locate. Such an initial scan would account for the failure to find superimposed serial position functions for the different list lengths as this scan would take longer, on the average, as list lengths increased. Given the added capability to move backward in STM by the retrieval of backward link information, the serial position functions of Figure 2 are less cryptic. For submemory-span lists (i.e., List Lengths 3 and 5), the self-terminating scan described by Sternberg (1967) may be the most efficient except for the final position probe at List Length 5 in the backward direction, where the use of a final item anchor point leads directly to the probe item. At List Length 7, where grouping occurs, the dips at Serial Position 4 for the forward probe condition and at Serial Position 5 for the backward probe condition are evidence for the use of the middle anchor point. Recency effects at the final point of the List Length 7 curves suggest use of the final item anchor point.

Although the three anchor point strategy described above is indicated by the data of subjects 1,2,3, and 5, there are other alternatives. For example, subject 6 did not report grouping of the longer lists, and his data indicate the use of only two anchor points with no middle curve dips (Figure 5). For subject 4 (Figure 3), neither the data nor the verbal report made it obvious what strategy he employed.

Overall, the present data suggest that the probe recall task may require several complex memory processes which are controlled by strategy level response schemes, especially when the sequences involved are not clearly subspan in length. The concepts of grouping, anchor points, and two scanning processes, one for presence and one to locate, are central to one information processing explanation of the data.

\section{REFERENCE NOTE}

Harris, G. J. Intertask explorations with a computer simulation model of short-term memory. Unpublished doctoral dissertation, State University of New York at Buffalo, 1970.

\section{REFERENCES}

Feigenbaum, E. A., \& Simon, H, A. A theory of the serial position effect. British Journal of Psychology, 1962, 53 , 307-320.

Laughery, K. R. Computer simulation of short-term memory: a component decay model. In G. H. Bower and J. T. Spence (Eds.), The psychology of learning and motivation: Advances in research and theory. Vol. 3, New York: Academic Press, 1969.

Murdock, B. B., Jr. Short-term retention of single paired-associates. Psychological Reports, 1961, 8, 280.

Murdock, B. B., Jr. Short-term retention of single paired associates. Journal of Experimental Psychology, 1963, 65, 433-443.

Murdock, B. B., Jr. The criterion problem in short-term memory. Journal of Experimental Psychology, 1966, 72, 317-324.

Murdock, B. B., Jr. Serial order effects in short-term memory. Joumal of Experimental Psy chology Monograph Supplements, $1968,76(4)$, part 2, 1-21.

Sternberg, $S$. Retrieval of contextual information from memory. Psychonomic Science, 1967, 8, 55-56.

Waugh, N. C., \& Norman, D. A. Primary memory. Psychological Review, 1965, 72, 89-104.

Yntema, D. B., \& Trask, F. P. Recall as search process. Joumal of Verbal Learning and Verbal Behavior, 1963, 2, 65-74

\section{NOTE}

1. The strategies described have been simulated (Harris, 1970) with an extended version of Laughery's (1969) computer simulation model of short-term memory. The reaction time predictions and curve shapes generated by the model provided good qualitative and quantitative fits with the experimental data.
(Received October 30, 1974 Revision received January $27,1974$. ) 